\title{
A human skin ultrasonic imaging to analyse its mechanical properties
}

\author{
Alexandre Delalleau*,** — Gwendal Josse** — Jérôme George** \\ Yassine Mofid*** — Frédéric Ossant*** _ Jean-Michel Lagarde**
}

* Laboratoire de Tribologie et Dynamique des Systèmes UMR 5513

ENISE, 58 rue Jean Parot F-42023 Saint-Etienne cedex 02

alexandre.delalleau@enise.fr

** Institut de Recherche Pierre Fabre, Centre de Recherche sur la Peau Hôtel Dieu Saint Jacques, 2 rue Viguerie, BP 3071 F-31025 Toulouse cedex 3 \{gwendal.josse,jean.michel.lagarde\}@pierre-fabre.com

*** Laboratoire UltraSons, Signaux et Instumentation, FRE 2448 CNRS Faculté de Médecine, 10 bd du tonnelé, BP 3223, F-37032 Tours cedex \{ossant,mofid\}@med.univ-tours.fr

ABSTRACT. The analysis of the skin mechanical behaviour is a key-point for different field of investigation. As the skin is a complex structure, studies are usually based on inverse methods that compare experimental and finite element numerical results. Besides the considered behaviour law, one of the most important question concerns the geometrical aspects of the skin tissue. In this paper, it is shown how high frequency ultrasound imaging helps the calculation of skin mechanical parameters. The hypodermis influence is firstly discussed through elastographic analyses. A specific procedure to measure the dermis thickness is then proposed to highlight that such a measurement must be considered to draw reliable conclusions. The obtained results are finally discussed to point out the interest of such simplifications for the study of more complex behaviour laws.

RÉSUMÉ. L'étude du comportement mécanique de la peau est primordiale pour différents champs d'expertise. Compte tenu de sa complexité, l'analyse du tissu cutané est généralement réalisée par méthodes inverses, proposant le couplage entre résultats expérimentaux et simulations par éléments finis. Outre le modèle de comportement à adopter, une question primordiale concerne la modélisation de la géométrie cutanée. Nous proposons d'utiliser ici des techniques ultrasonores afin d'établir une géométrie simplifiée de la peau. L'influence de l'hypoderme est tout d'abord discutée à l'aide d'observations élastographiques. Une technique de mesure de l'épaisseur du derme est ensuite présentée afin de mettre en exergue son importance pour l'obtention de résultats exacts. Nous concluons finalement cette analyse en soulignant la conséquence de telles simplifications sur l'étude de modèles de comportement plus complexes.

KEYWORDS: skin, finite elements, elastography, ultrasound, inverse method, behaviour.

MOTS-CLÉS : peau, éléments finis, élastographie, échographie, méthode inverse, comportement.

DOI:10.3166/EJCM.18.105-116 @ 2009 Lavoisier, Paris

EJCM - 18/2009. Numerical models in biomechanics, pages 105 to 116 


\section{Introduction}

The skin is composed of three main layers: the epidermis, the dermis and the hypodermis. The epidermis is the outer layer of skin. It possesses a thickness which varies from $100 \mu \mathrm{m}$ to $1.5 \mathrm{~mm}$ at palms and soles and is also divided into five layers including the well-known stratum corneum. The mechanical properties of the epidermis and the stratum corneum are frequently discussed in literature (Agache, 2000). Most of the studies show that the temperature and the relative humidity strongly affect both the thickness and the mechanical properties of the stratum corneum (Papir et al., 1975). Its Young's modulus $E$ can vary from 57.8MPa (Panisset et al., 1994) to 1GPa (Pailler-Mattei, 2004), respectively for suction and indentation tests performed on the volar aspect of the forearm. The hypodermis is the lowermost layer of the skin. It is composed of fat cells (adipocytes) and links the dermis to the subcutaneous structures such as the muscles. As it presents low mechanical properties this layer is usually studied through hyperelastic behaviour laws. The identified elastic modulus presents values from $0.02 \mathrm{kPa}$ (Hendricks et al., 2003) to $8.2 \mathrm{kPa}$ (Tran et al., 2005). The dermis is known to be mainly responsible for the mechanical properties of the skin. It is composed of collagen and elastin fibres, which present linear elastic properties (Wilkes et al., 1973), floating in a matrix of proteoglycans, which is a viscous fluid (Oomens et al., 1987). The interaction between these components gives the skin a complex mechanical behaviour which is known to be nonlinear, viscoelastic, anisotropic and quasi-incompressible (Delalleau et al., 2007a).

Hence, skin is a composite-like structure which shows a very complex mechanical behaviour. As it is a living structure, it has to be studied in vivo and this leads to difficult analyses of its mechanical properties which are of great interest for different field of investigation such as dermatology, surgery and cosmetology. Inverse methods, whose purpose is to compare experimental and numerical results, are usually used for that pupose. Numerical studies are based on several assumptions including the geometry and the mechanical behaviour of the skin which is widely discussed in literature (Delalleau, 2007). In contrast, few studies account for the anisotropic and viscous aspects of the skin (Khatyr et al., 2004) and most of the analyses are based on nonlinear elastic behaviour laws (Delalleau et al., 2007a; Hendricks et al., 2003). The proposed behaviours present the advantage of being easy to use to study the effect of a medical treatment on the skin mechanical properties. Actually, owing to the numerous curves to be analysed, numerical models must be not only as simple but also as relevant as possible. Nevertheless, besides the considered behaviour law, one of the most important questions is related to the geometrical aspects of the skin tissue. Actually, the skin structure is usually considered as a single layer (Delalleau et al., 2007a; Hendricks et al., 2003; Kathyr et al., 2004) however only a few papers argue this assumption.

This paper first aims at proving that the influence of the hypodermis can be neglected. This analysis is based on comparisons between echographic observations of the suction test (Diridollou et al., 1998) and finite elements models of a threelayer structure. Calculations are then performed on semi-infinite and single layers to 
conclude on the best model to use. An order of magnitude of the hypodermis elastic modulus is given. The influence of the ratio between the thicknesses of the dermis and the hypodermis is then discussed. This points out that the thickness of the dermis has to be accurately measured to obtain reliable results. A specific procedure based on active contour imaging techniques is presented (Lagarde et al., 2005). Theoretical aspects on the influence of the measured thickness on the identified mechanical parameters are then proposed. The obtained results are finally discussed and an extension of this work to more complex problems is mentioned.

\section{General aspects}

\subsection{Experimental test}

Various experimental tests have been designed to identify the mechanical properties of the skin in vivo (Agache, 2000). The presented results are related to the suction test which is performed on the volar aspect of the forearm (Diridollou et al., 1998). This experiment consists in applying a negative pressure at the skin surface using a Cutometer CM570 (Courage and Khazaka, Cologne, Germany). The skin is sucked into an aperture (6mm diameter) and forms a dome whose deflection is recorded for each step of pressure. To identify the skin mechanical properties, an inverse method being used to compare experimental and finite element results of the suction test is proposed (Delalleau et al., 2007b).

\subsection{Finite element calculations}

The numerical simulations are performed with the Systus ${ }^{\mathrm{TM}}$ finite element software. The layers of the skin are considered to be homogeneous. Owing to the low stress rate, a quasi-static option is used for the calculations. The geometry is considered to be axisymmetrical. The suction test usually involves large deformations and large displacements in the skin. Hence, the behaviour law and the formulation that are used should account for the geometrical nonlinearity of the material (Delalleau et al., 2007a). The skin layers are thus modelled by using hyperelastic neo-Hookean laws as suggested by Hendricks (Hendricks et al., 2006). The corresponding potential $\boldsymbol{W}$ (Treloar, 1947) is described by:

$$
\boldsymbol{W}=X_{1}\left(I_{1}-3\right)
$$

where $X_{1}$ is the elastic modulus, and $I_{1}$ is the first invariant of the right CauchyGreen deformation tensor $\mathbf{C}$. This potential is incompressible and a Poisson ratio of 0.49 is artificially considered. To avoid the volumetric locking, second order elements with a reduced integration scheme are used. 


\section{The hypodermis layer}

\subsection{Method}

Most of the studies that aim at identifying the mechanical properties of the skin are usually based on single-layer models. Actually, results from literature generally state that the hypodermis presents low elastic properties (Samani et al., 2001; Diridollou, 1994). Hence, the effect of sub-dermal structures can be neglected (Cook et al., 1977; Diridollou et al., 2000; Hendrick et al., 2003). Cook et al., (1977) have compared in vivo and ex vivo suction measurements on rat skin and has clearly stated that there is only an effect of subcutaneous tissue attachment for low strains. This leads to conclude that the hypodermis limits the subcutaneous structures stresses. (Diridollou et al., 2000) and (Hendricks et al., 2003), have observed the dermis and the hypodermis deformations during a suction experiment. The dermis presents high displacements but small strains whereas the underlying tissues do not move. To draw a reliable finite element model of the skin and to identify its mechanical properties, (Hendricks et al., 2003) have proposed a 2-layer structure (dermis/hypodermis). Elastic moduli of $50 \mathrm{kPa}$ and $0.02 \mathrm{kPa}$ were respectively identified for the dermis and the hypodermis. Nevertheless this result has to be compared with the study of (Samani et al., 2003) related to the adipose tissue of the breast which presents a Young's modulus of $1.9 \mathrm{kPa}$ (for small strains: $E=6 X_{1}$ ). Finally, (Tran et al., 2005) have identified an elastic modulus of $8.2 \mathrm{kPa}$ using an indentation device. These results state that the identification of the mechanical properties of the hypodermis is an open debate.

However, an order of magnitude of the elastic modulus of the hypodermis can be proposed while comparing ultrasounds images and finite elements models of the suction test. A three-layer structure is studied. The first layer is related to the dermis and the epidermis and shows a thickness of $1 \mathrm{~mm}$. The second layer models a $1 \mathrm{~mm}$ hypodermis. Finally, the third layer, which stands for a bone, is artificially modelled thought restrained boundary conditions of the nodes corresponding to the lower part of the hypodermis. A neo-Hookean behaviour (see Equation [1]) is used to model both the layers. The elastic modulus of the dermis is chosen as $X_{1}^{\mathrm{d}}=80 \mathrm{kPa}$ in order to obtain reliable values for the deflection measurements. A range of values from $X_{1}^{h}=10^{-2} \mathrm{kPa}$ to $X_{1}^{h}=10 \mathrm{kPa}$ is considered to account for the results previously presented. The suction pressure is applied up to 200mbar. The obtained results are compared with a single-layer and a semi-infinite models as well for $X_{1}=80 \mathrm{kPa}$.

\subsection{Results}

Figure 1 plots the effect of the hypodermis elastic modulus on the maximal deflection (for $\mathrm{p}=200 \mathrm{mbar}$ ). It clearly states that, for high values of $X_{1}^{h}$, the results tend to the semi-infinite deflection whereas for lower values, a single-layer assumption can be made. One can remark that, due to the specific boundaries 
conditions at the hypodermis/bone interface, the 2-layer model presents a lower maximal deflection than the one proposed by the semi-infinite substrate.

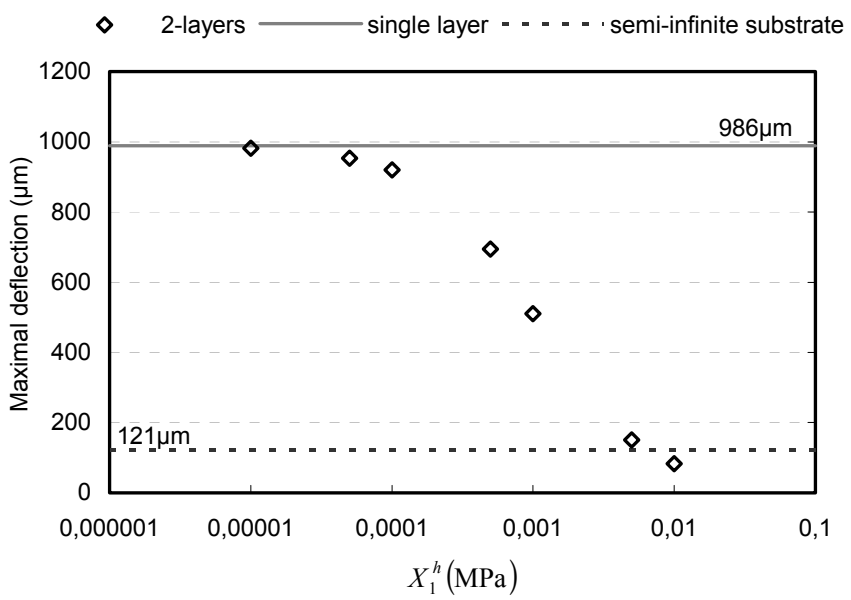

Figure 1. Effect of the elastic modulus of the hypodermis on the maximal deflection $(p=200 m b a r)$

The hypodermis Young's modulus thus influences the obtained results and its value must be discussed. One can consider the specific high-frequency static elastography techniques developed by Mofid (Mofid et al., 2004). It is based on a $20 \mathrm{MHz}$ device (Dermcup 2020 ${ }^{\mathrm{TM}}$, GIP Ultrasouns, Tours, France) which is used to scan the skin during a stress. This analyse was previously performed through an extensometer (Mofid et al., 2006) and was recently extended to the suction test (Ossant et al., 2006). Ultrasound elastograms are computed through intercorrelation of the radio-frequency lines between two successive images. Axial strain field are calculated all along the test (Mofid et al., 2004).

The obtained images (Figure 2) show, during the suction test, that the hypodermis strongly deforms whereas the dermis only moves vertically. Numerical calculations can correlate such results considering $X_{1}^{\mathrm{d}}=30 \mathrm{kPa}, X_{1}^{h}=10^{-2} \mathrm{kPa}$ and a thickness of the dermis equals to $1.7 \mathrm{~mm}$ (see Figure 2). The computed maximum deflection is about $0.50 \mathrm{~mm}$ whereas the experimental one equals $0.54 \mathrm{~mm}$. The axial strains in the dermis and the hypodermis can also be compared. A maximum axial strain component about $30-35 \%$ was measured through elastographic images (see Figure $2 a$ ) and the numerical calculations present a value about $40 \%$ (see Figure $3 \mathrm{a}$ ). When using the hypodermis elastic modulus identified by (Tran et al., 2004) one can notice that the actual axial strain is much below the experimental one (see Figure $3 b$ ) and the deflection presents non reliable values. This was successfully checked for different values of $X_{1}^{\mathrm{d}}$. 
a)

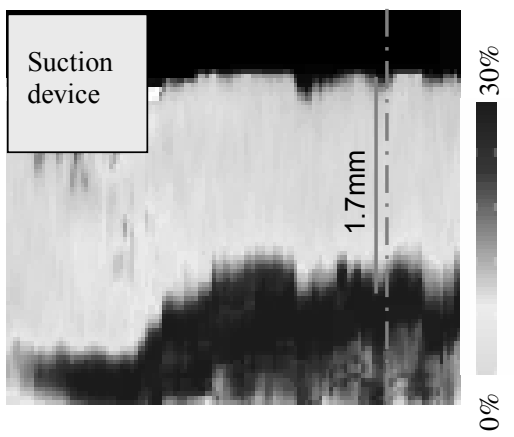

b)

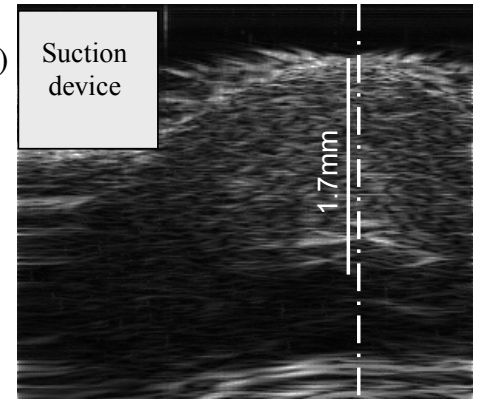

Figure 2. Images obtained for a suction test performed on the volar aspect of the forearm. Elastographic (a) and echographic (b) images for $p=100 \mathrm{mbar}$

Nevertheless such a result should be discussed according to the dermal thickness, its elastic modulus and the one related to the hypodermis. Figure 4 plots the variations of the maximum deflection for different combinations of the dermis thickness, for $X_{1}^{\mathrm{d}}$ and for $X_{1}^{\mathrm{h}}$. For every case study, reliable results (which correspond to suction experiments) can be obtained for low values of the hypodermis elastic modulus. As previously observed (see Figure 1), for such values $X_{1}^{\mathrm{h}}$ barely affects the results. $X_{1}^{\mathrm{d}}$ presents a restrained influence on the deflection whereas the dermal thickness appear to be one of the major criteria for the skin modelling. Hence, it has to be precisely measured to avoid identification errors and this requires the use of a specific imaging technique (Lagarde et al., 2005).

\section{Dermis thickness measurements}

\subsection{Method}

As presented in the above section, the thickness of the dermis appears to be one of the most important parameters for the skin modelling. Studies used to consider its mean value, measured by using histological analysis. Two major problems are then highlighted. First, results from literature clearly state that the thickness of the skin strongly differs according to the sex and the age of an individual (Agache, 2000). While considering a mean value, identification estimates are thus done. The second aspect is related to the cosmetic analysis. As the dermis stiffness and its thickness both influence the measurements (see Figure 4), it is not possible to distinguish a thickness influence from the stiffness modification which is generally expected. A specific automatic measurement of the dermal thickness was thus developed by (Lagarde et al., 2005). 
a)

b)

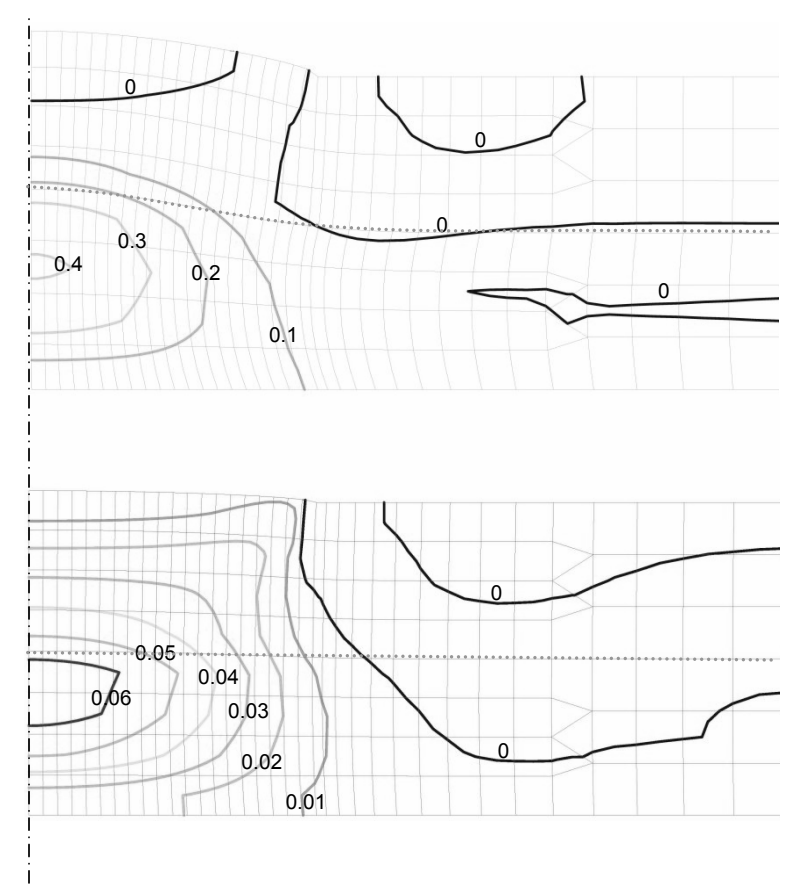

Figure 3. Axisymmetrical section of the axial strain component for finite element models of the suction test. a) $X_{1}^{\mathrm{d}}=30 \mathrm{kPa}, X_{1}^{h}=10^{-2} \mathrm{kPa}$. b) $X_{1}^{\mathrm{d}}=30 \mathrm{kPa}$, $X_{1}^{h}=10 \mathrm{kPa}(p=100 \mathrm{mbar})$

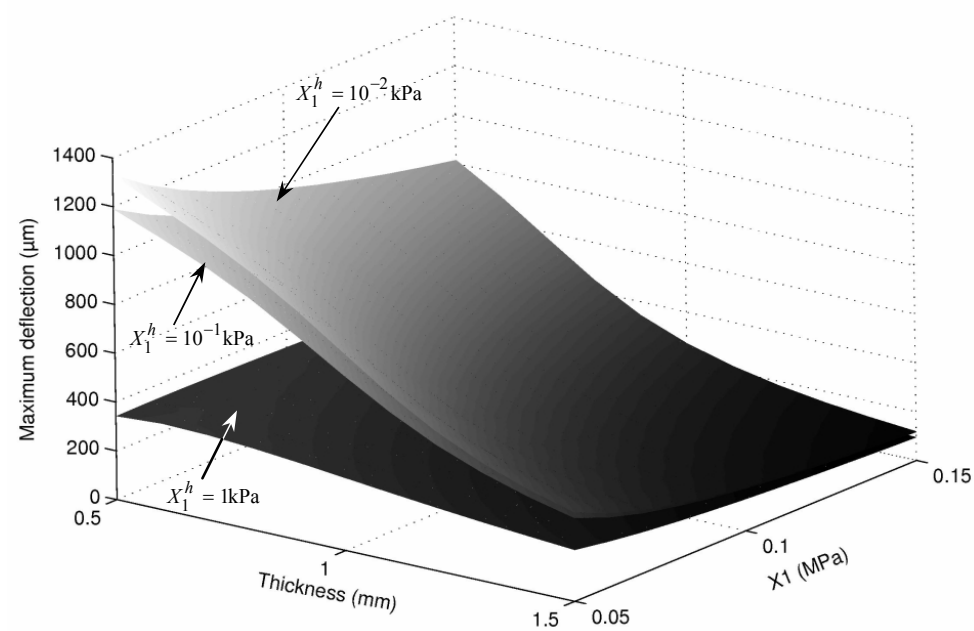

Figure 4. Maximum deflection for $p=100 \mathrm{mbar}$ according to the thickness and the stiffness of the dermis. The results are presented for different values of $X_{1}^{h}$ 
This technique is based on an active contour algorithm which is used to treat $20 \mathrm{MHz}$ B-scan mode Dermcup ${ }^{\mathrm{TM}}$ images. Its main advantages are to avoid interoperator variability (Lagarde et al., 2005) and to obtain results rapidly. Indeed, the time required by a specialist is usually about 5-10min per image whereas the computation time is usually about $1.5 \mathrm{~s}$.

The active contours consist of initializing a curve near the contour to be defined and of using an iterative process to fit it. This technique, which was initially developed by Kass (Kass et al., 1987), was called the snake. It is defined through a parametered curve $v(s)$ :

$$
\begin{aligned}
& \Omega:[0,1] \rightarrow \Re^{2} \\
& s \mapsto v(s)=(x(s), y(s))
\end{aligned}
$$

where $s$ is the curvilign abscissa and $x$ and $y$ its corresponding cartesian coordinates. The deformation of the snake is then evolved so as to minimize the energy $E(v)$ :

$$
v \mapsto E(v)=\int_{\Omega}\left[\alpha(s)\left\|v_{s}(s)\right\|^{2}+\beta(s)\left\|v_{s s}(s)\right\| \|^{2}+P(v(s))\right] d s
$$

where $v_{s}$ and $v_{s s}$ are respectively the first and second order derivatives of $v(s)$, and the parameters $\alpha(s)$ and $\beta(s)$ are related to the elasticity and the bending stiffness of the snake. $P(v(s))$ is related to the external constrains of the problem.

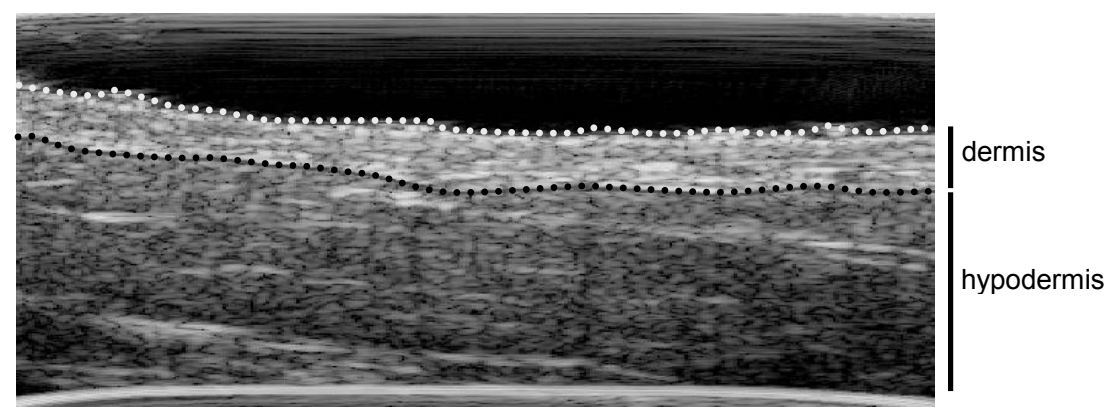

Figure 5. Upper and lower contours of the dermis using a 20MHz B-scan imaging of the forearm skin

This standard approach is related to closed contours. Hence, according to the obtained images (see Figure 5), it cannot be used to evaluate the dermal thickness. This technique was thus extended to non-closed contours (Lagarde et al., 2005). 
Each snaxel (snake/pixel) is usually affected by his own weight, the one of its immediate neighbours and the one of its neighbours' neighbours. Specific weights (i.e. virtual snaxels) were added to the first and last snaxels and their previous neighbours' weights were suppressed to open the contour. To avoid instabilities of the snake, the lateral displacements of its extremities were then restrained. Finally, as the orientation of the contours has to be considered, a second order derivation of the image is used for the calculations, and a local orientation of the snake is calculated by using the two immediate neighbours of the considered snaxel.

\subsection{Results}

Figure 5 gives an example of the obtained contours for a 20MHz B-scan imaging performed on the forearm. They closely fit the lower and upper parts of the dermis. This image could be the starting point of the numerical simulations, however, to simplify the geometry of the finite element model, the dermis is considered to be a constant thickness plane. This thickness $e^{d}$ is calculated as follows:

$$
e^{d}=\frac{1}{L} \int_{l}\left(\mho_{l}-\mho_{u}\right) d l
$$

where $L$ is the image length and $\mathscr{C}_{1}$ and $\mathscr{C}_{u}$ are respectively the dermis upper and lower contours. This automatic procedure was finally compared to manual measurements performed on 612 images (Lagarde et al., 2005). Considering the fully-automatic algorithm, a correlation coefficient about 0.47 was pointed out. As the manual calculations are assumed to provide the best results, the obtained thicknesses should be discussed. Indeed, according to the site, the dermis/hypodermis interface may not appear clearly. Hence, the initial conditions of the calculation may lead the snake to fit non-reliable contours. New initial conditions were thus manually specified for $18 \%$ of the images. The obtained correlation coefficient about 0.94 proves the interest of such a method.

The influence of an error measurement of the dermal thickness on the identified mechanical parameters can also be estimated. Figure 6.a plots the variations of the maximum deflection for $\mathrm{p}=200 \mathrm{mbar}$ according to the thickness of a single layer model. A neo-Hookean behaviour law with $X_{1}=80 \mathrm{kPa}$ and $v=0.49$ is considered. One can note that according to Figure 4, Figure 6.a shows a high variation of the deflection for values of the thickness from $0.5 \mathrm{~mm}$ to $1.5 \mathrm{~mm}$. This indicates that, for such thicknesses, a measurement error will strongly affect the identified mechanical parameters. While considering for example a $1 \mathrm{~mm}$-thick model with $X_{1}=80 \mathrm{kPa}$, the identified elastic modulus for a thickness of $1.25 \mathrm{~mm}(0.25 \mathrm{~mm}$ measurement error) is $X_{1}=54 \mathrm{kPa}$ (see Figure 6.b). Hence, for such a thickness, a $25 \%$ measurement error induces a $32 \%$ identification miscalculation. This clearly highlights that the thickness measurements should be carefully performed. One can 
finally note that literature analyses (Agache et al., 1992) (Diridollou et al., 1994) (Khatyr et al., 2004) are generally based on initial measurements of the dermis thickness. Hence, its variation during the experiment, which affects the calculation, is not considered contrary to finite elements simulations.
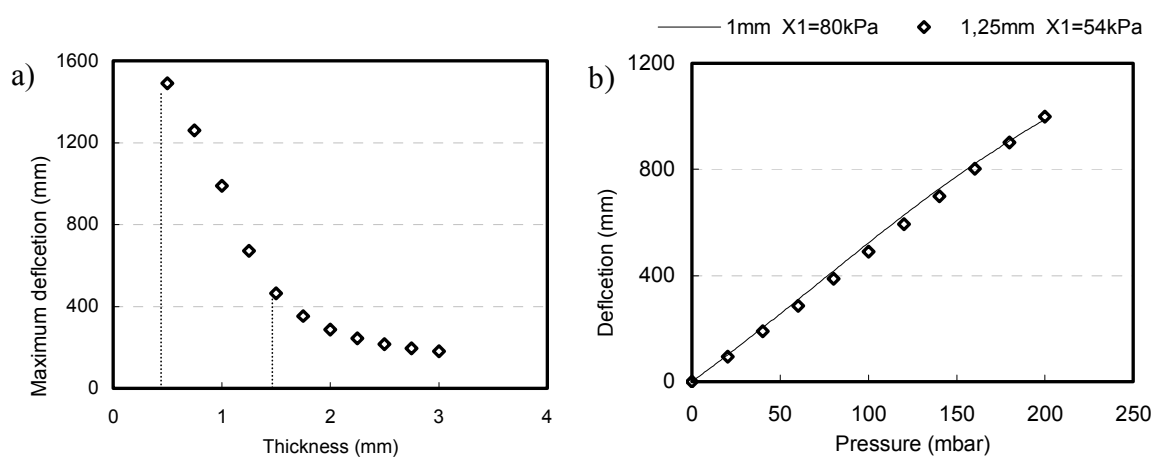

Figure 6. a) Influence of the thickness of the single layer model on the maximum deflection ( $\left.p=200 \mathrm{mbar}, X_{1}=80 \mathrm{kPa}\right)$. b) Comparison between the test-curve (1mmthick model with $X_{1}=80 \mathrm{kPa}$ ) and the identified one for a $1.25 \mathrm{~mm}$-thick model $\left(X_{1}=54 \mathrm{kPa}\right)$

\section{Discussion}

This paper aimed at proving the interest in using the medical imaging to model the skin mechanics. High-frequency static elastography techniques developed by Mofid were for example used to study the influence of the hypodermis during a suction experiment. The obtained results prove that the dermis mainly affects the deflection and they state about the range of the hypodermis elastic moduli proposed by literature. Finally, according to this analysis, the complex three-layer structure of the skin can be simplified to a single layer whose thickness seems obviously to be one of the most significant parameters for the skin modelling. A specific measurement of this thickness was thus proposed by Lagarde to improve the rapidity and the accuracy of the existing manual method. In fact, an error on the dermis thickness measurement usually induces an even highest miscalculation of the identified mechanical parameters. Hence, accurate methods and devices are required to study media such as the skin. Nevertheless, the interests in the skin imaging can be improved while considering more complex problems. The boundary conditions of the hypodermis/dermis and the hypodermis/subcutaneous structures interfaces are for example of primary importance to draw reliable conclusions on the hypodermis influence on the measured deflection. Moreover, the specific mechanical behaviour of this layer can be assessed by using elastographic imaging. Finally, such a technique may provide major informations for the mechanical parameters identification. Displacements and strains fields can for 
example be considered instead of a standard analysis (e.g. deflection versus pressure curve). This may provide more stable and reliable optimization computations. Simple static imaging can also be used to accurately define the individual dermal geometry. Actually, numerical models are currently based on 2D single plane layers however Figure 5 clearly shows that the dermis contours are not straight. Three dimensional imaging may also be an interesting study point to simulate how the skin structures move along each others and thus to understand better skin mechanics. Nevertheless, analyses should account first for individual measurements of the dermal thickness and for more complex behaviour laws.

\section{References}

Agache P., Varchon D., Rochefort A., Humbert P., 'Non invasive assessement of biaxial Young's modulus of human skin in vivo", Journal of Biomechanics, vol. 25, 1992, p. 115-120.

Agache P., Physiologie de la peau et explorations fonctionnelles cutanées, Collection explorations fonctionnelles humaines, 2000.

Cook T., Alexander H., Cohen M., "Experimental method for determining the 2-dimensional mechanical properties of living human skin”, Medical and Biological Engineering and Computing, vol. 15, 1977, p. 381-390.

Delalleau A., Josse G., Lagarde J.-M., Zahouani H., Bergheau J.-M., "A nonlinear elastic behaviour to assess the mechanical parameters of human skin in vivo", Skin Research and Technology, accepted July 2007a, available online.

Delalleau A., Josse G., Lagarde J.-M., Zahouani H., Bergheau J-M., "Use of the Kalman Filters for the analysis of the mechanical properties of human skin in vivo", Inverse Problems in Science and Engineering, accepted May 2007b.

Delalleau A., Analyse du comportement mécanique de la peau in vivo, $\mathrm{PhD}$. Thesis, Jean Monnet University, Saint-Etienne, France, 2007.

Diridollou S., Berson M., Vabre V., Black D., Karlsson B., Auriol F., Gregoire J.-M., Yvon C., Vaillan L., Gall Y., Patat F., "An in vivo method for measuring the mechanical properties of the skin using ultrasound", Ultrasound in Medecine and Biology, vol. 24, $\mathrm{n}^{\circ} 2,1998$, p. $215-224$.

Diridollou S., Patat F., Gens F., Vaillant L., Black D., Lagarde J-M., Gall Y., Berson M., "In vivo model of the mechanical properties of the human skin under suction", Skin Research and technology, vol. $6, \mathrm{n}^{\circ} 21,2000$, p. 221-225.

Hendricks F.M., Brokken D., van Eemeren J.T.W., Oomens C.W.J., Baijens F.P.T., Horsten J.B.A.M., "A numerical-experimental method to characterize the non-linear mechanical behaviour of human skin”, Skin Research and Technology, vol. 9, 2003, p. 274-283.

Hendricks F.M., Brokken D., Oomens C.W.J., Bader D.L., Baijens F.P.T., « The relative contributions of different skin layers to the mechanical behaviours of human skin in vivo using suction experiments ", Medical Engineering and Physics, vol. 28, 2006, p. 259-266. 
Kass M., Witkin A., Terzopoulos D., "Snakes: active contour model”, International Journal of Computer Vision, 1987, vol. 1, p. 321-331.

Khatyr F., Imberdis C., Vescovo P., Varchon D., Lagarde J-M., "Model of the viscoelastic behaviour of skin in vivo and study of anisotropy”, Skin Research and Technology, vol. 10, 2004, p. 96-103.

Lagarde J.-M., George J., Soulcié R., Black D., “Automatic measurement of dermal thickness from B-scan ultrasound images using active contours", Skin Research and Technology, vol. $11, \mathrm{n}^{\circ} 2,2005$, p. 79-90.

Mofid Y., Ossan F., Imberdis C., Patat F., "New elastographic algorithm for in vivo study of mechanical behaviour of skin", Proceedings of the international conference on Ultrasounds Measurement and Imaging of Tissue Elasticity, 2004, p. 59.

Mofid Y., Ossant F., Imberdis C., Josse G., Patat F., "In vivo imaging of the skin under stresses: Potential of high-frequency (20MHz) static 2-D elastography", IEEE Transactions on Ultrasonics, Ferrolectrics and Frequency Control, vol. 53, n 5, 2006, p. 925-935.

Oomens C.W.J., van Campen D.H., Grootenboer H.J., "A mixture approach to the mechanics of skin”, Journal of Biomechanics, vol. 20, n 9, 1987, p. 877-885.

Ossant F., Patat F., Mofid Y., Gahagnon S., Josse G., "In vivo high frequency elastography for mechanical behaviour of human skin under suction stress: elastograms and kinetics of shear, axial and lateral strain fields", IEEE Ultrasonics Symposium, 2006, p.1041-1044.

Pailler-Mattéi C., Caractérisations mécanique et Tribologique de la peau humaine in vivo, PhD Thesis, Ecole Centrale de Lyon, France, 2004.

Panisset F., Varchon D., Agache P., "Non invasive measurement of stratum corneum Young's modulus in vivo", XIV th Congress on Biomechanics, Paris, 1994.

Papir Y.S., Hsu H-H., Wildnauer R.H., "The mechanical properties of stratum corneum. I. The effect of water and ambient temperature on the tensile properties of newborn rat stratum corneum", Biochimica et Biophysica acta, vol. 399, 1975, p. 170-180.

Samani A., Bishop J., Yaffe M., Plewes D.B., "Biomechanical 3-D Finite Element Modeling of the Human Breast for MR/X-ray using MRI Data", IEEE Transactions on Medical Imaging, vol. 20, $\mathrm{n}^{\circ} 4,2001$, p. 271-279.

Samani A., Bishop J., Luginbuhl C., Plewes D.B., "Measuring the elastic modulus of ex vivo small tissue sample", Physics in Medicine and Biology, vol. 48, 2003, p. 2183-2198.

Tran H.V., Charleux F., Ehrlacher A., Ho Ba Tho M-C., «Propriétés mécaniques multicouches de la peau humain in vivo, modèle numérique anatomique ", Proceedings of the $7^{e}$ Colloque National en Calcul des Structures de Giens, 2005.

Treloar L.R.G., "Stresses and birefringence in rubber subjected to general homogeneous strain”, Proceedings of the Physical Society in London, vol. 60, p. 135-144, 1947.

Wilkes G.L., Brown I.A., Wildnauer R.H., "The biomechanical properties of skin", Critical Reviews in Bioengineering, vol. 1, $\mathrm{n}^{\circ}$ 4, 1973, p. 453-495. 